\title{
Regular and Moderate Exercise Counteracts the Decline of Antioxidant Protection but Not Methylglyoxal-Dependent Glycative Burden in the Ovary of Reproductively Aging Mice
}

\author{
S. Falone, ${ }^{1}$ S. Jr Santini, ${ }^{1}$ V. Cordone, ${ }^{1}$ M. Grannonico, ${ }^{2}$ M. Cacchio, ${ }^{3}$ \\ G. Di Emidio, ${ }^{1}$ C. Tatone, ${ }^{1}$ and F. Amicarelli ${ }^{1,4}$ \\ ${ }^{1}$ Department of Life, Health and Environmental Sciences, University of L'Aquila, L'Aquila, Italy \\ ${ }^{2}$ Department of Microbiology and Immunology, Albert Einstein College of Medicine, Bronx, NY, USA \\ ${ }^{3}$ Department of Biomedical Sciences, "G. d'Annunzio" University, Via dei Vestini, Chieti Scalo, Italy \\ ${ }^{4}$ Institute of Translational Pharmacology (IFT), National Research Council (CNR), L’Aquila, Italy
}

Correspondence should be addressed to S. Falone; stefano.falone@univaq.it

Received 9 October 2016; Revised 17 November 2016; Accepted 7 December 2016

Academic Editor: Mauricio Krause

Copyright (C) 2016 S. Falone et al. This is an open access article distributed under the Creative Commons Attribution License, which permits unrestricted use, distribution, and reproduction in any medium, provided the original work is properly cited.

\begin{abstract}
Population aging results in urgent needs of interventions aimed at ensuring healthy senescence. Exercise often results in healthy aging, yet many molecular mechanisms underlying such effects still need to be identified. We here investigated whether the agedependent accumulation of oxidative and methylglyoxal- (MG-) related molecular damage could be delayed by moderate exercise in the mouse ovary, an organ that first exhibits impaired function with advancing age in mammals. CD1 female mice underwent twoor four-month treadmill-based running through the transition from adult to middle age, when ovaries show signs of senescence, and markers of protection against reactive oxygen species (ROS) and MG were measured. The long-term exercise reduced the protein oxidative damage in the ovaries $(P<0.01)$, and this was linked to the preservation of the glutathione peroxidase protection against ROS $(P<0.001)$, as well as to the increased glutathione availability $(P<0.001)$. Conversely, even though the age-related deactivation of the MG-targeting systems was partially prevented by the long-term running programme $(P<0.001)$, exercised mice were not protected from the age-dependent glycative burden. In summary, lately initiated regular and moderate exercise limited some changes occurring in the ovaries of middle-aged mice, and this might help to develop nonpharmacological cointerventions to reduce the vulnerability of mammalian ovaries towards redox dysfunctions.
\end{abstract}

\section{Introduction}

In modern advanced societies, population aging and life expectancy are constantly increasing, leading to major ageassociated health issues in both sexes. Women, who generally live longer than men, will spend nearly a third of their lifetime in postmenopause. In women, menopause indicates the absolute end of reproductive life. However, a decline in fertility is already apparent 20 years before menopause, and 10 years before menopause the ability to conceive is extremely low [1]. Ovarian aging is a major determinant of this agedependent decrease in female fertility and is related to a decrease in the size of the ovarian follicle pool and the quality of the oocytes therein [2], as well as to alterations of the ovarian stroma [3]. Female reproductive aging is associated with some disorders known to increase in mid-life including fetal aneuploidy, cognitive impairment, and cardiovascular disease [4]. Hence, how to maintain the reproductive health and improve the ovarian lifespan is currently considered as an area of great interest.

Impaired defence against cytotoxic reactive oxygen species (ROS) and methylglyoxal (MG), a powerful endogenous glycating and prooxidant compound, seems to be critically linked to aging $[5,6]$. The ovary is the first organ to show signs of physiological aging and is among the most vulnerable organs to age-dependent glycative and oxidative burden 


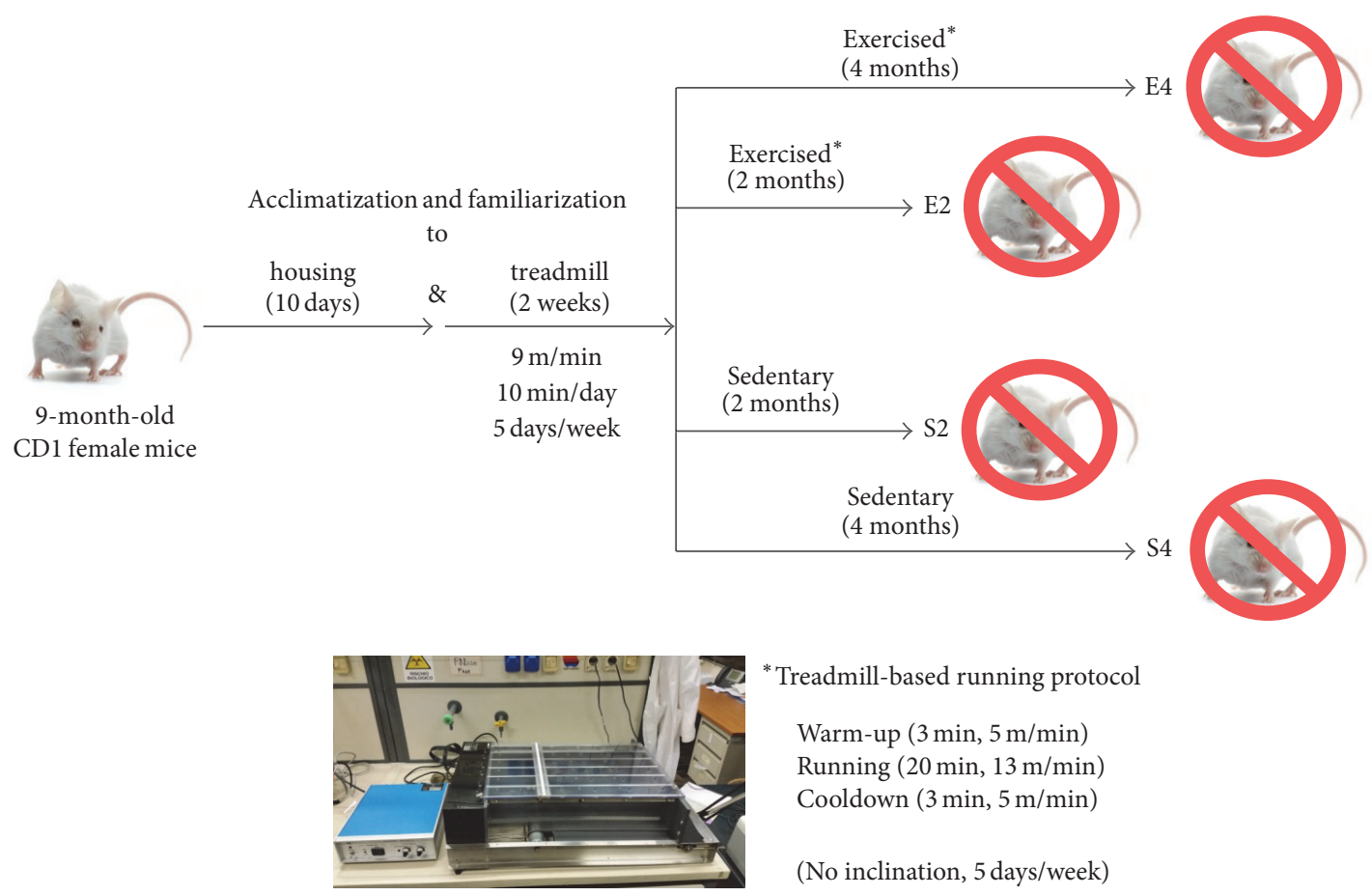

Figure 1: Experimental design.

[2, 7-11]. Accordingly, mice that exhibit impaired biosynthesis of glutathione (GSH), the most abundant thiol-containing intracellular antioxidant and essential cofactor for antioxidant and antiglycative enzymes, such as glutathione peroxidase and glyoxalase 1 , show accelerated ovarian aging due to increased ovarian oxidative stress [12].

Both ROS- and MG-dependent molecular damage have been hypothesized to be downregulated by simple lifestylebased interventions, among which physical exercise is emerging as the leading determinant of healthy aging [13]. Recently, it has been reported that reproductive dysfunctions in mice with polycystic ovary syndrome (PCOS), an ovarian disease associated with glycative stress $[11,14]$, can be ameliorated by exercise without weight loss [15].

In this context, we previously reported that long-term moderate running elicits important antiaging protective effects in the hippocampal formations and brain cortex of middle-aged mice, mainly through the activation of the major ROS- and MG-targeting enzymatic systems [16, 17].

On this basis, the question as to whether physical exercise could help to preserve antioxidative and antiglycative defences in the aging ovary may be raised. Despite the emerging interest in this field, specific studies are currently unavailable.

We here investigated whether the age-dependent disturbance of oxidative and methylglyoxal- (MG-) related metabolism could be affected by moderate exercise in the mouse ovary, a mammalian organ that exhibits impaired function with advancing age.

In order to test our hypothesis, reproductively aging female CD1 mice underwent a moderate treadmill-based running programme initiated during the transition from adult to middle age, a period in which ovaries already exhibit signs of senescence, in terms of decline of both ovarian reserve and oocyte quality [18-20].

Ovaries were processed for the assessment of the activity of enzymatic systems responsible for the protection against oxidative and methylglyoxal-induced molecular damage, as well as for the level of expression of Anti-Müllerian hormone $(\mathrm{AMH})$, an excellent marker of ovarian reserve [21, 22].

\section{Materials and Methods}

2.1. Animals and Running Protocol. Nine-month-old CD1 female mice (45-50 g; $N=48$; Harlan Laboratories, Inc., Frederick, MD, USA) were acclimatized for 10 days in the laboratories of the Excellence Research Centre on Aging of the University Foundation "G. d'Annunzio" of Chieti (Italy) to the housing conditions $\left(22 \pm 2^{\circ} \mathrm{C}, 12-12 \mathrm{~h}\right.$ light-dark cycle, with lights on from 8 a.m. to 8 p.m., free access to water and food, 6 animals per cage). The familiarization and running protocols used in this research were already been described in previous works $[16,17,23]$. This exercise was recognized as moderate based on the intensity at $65 \%$ to $70 \%$ of the maximal oxygen uptake [24]. Briefly, the animals were familiarized to the Exer 3/6 motorized low-noise treadmill (Columbus Instruments, Columbus, $\mathrm{OH}$, USA) for 2 weeks ( $9 \mathrm{~m} / \mathrm{min}$ for $10 \mathrm{~min}, 5$ days/week). Mice were randomly assigned to four groups (12 mice each): mice undergoing a treadmill-based running programme for either 2 or 4 months (E2 and E4, resp.), and mice undergoing a sedentary regimen for either 2 or 4 months (S2 and S4, resp.) (Figure 1).

Exercised groups underwent the running programme (warm-up at $5 \mathrm{~m} / \mathrm{min}$ for $3 \mathrm{~min}$, running at $13 \mathrm{~m} / \mathrm{min}$ for 
$20 \mathrm{~min}$, and cooldown at $5 \mathrm{~m} / \mathrm{min}$ for $3 \mathrm{~min}$, zero inclination, 5 days/week), starting from $10 \mathrm{~min} /$ day and reaching the final work load by incrementing $1 \mathrm{~min}$ every day (Figure 1). We used the most common running protocol employed in investigations where low-intensity exercise was needed [25, 26]. The moderateness of the exercise model was confirmed also by the fact that the treadmill was operating at zero inclination; therefore the exercise effort was strongly reduced, as the mice needed only to move forward, but not to climb. Moreover, the "running" protocol consisted of a fast walk; in fact at least two limbs of the animals were in contact with the ground while exercising. In order to avoid unnecessary stress, mice were motivated to run only by gentle hand prodding. Sedentary groups were exposed to the same environmental stimuli (handling, treadmill motor noise, and vibration and deprivation of food and water) while exercising subjects were running. Animal weight and food consumption were monitored daily throughout the experiment. One day after the last running session, mice were sacrificed by rapid decapitation, and ovaries were removed, immediately frozen in liquid $\mathrm{N}_{2}$, and stored at $-80^{\circ} \mathrm{C}$. All possible measures were taken to reduce the number and the suffering of animals, in accordance with the European Union Directive 2010/63/EU for animal experiments.

\subsection{Ovary Extract Preparation for Enzymatic Activity Assess-} ments. Ovaries were lysed (300 mg/mL) in either (a) $100 \mathrm{mM}$ phosphate buffer ( $\mathrm{pH} 7$ ), containing $1.5 \mathrm{mM}$ dithiothreitol (DTT) and $1 \mathrm{mM}$ EDTA (for glyoxalase 1, glyoxalase 2, and glutathione peroxidase) or (b) $100 \mathrm{mM}$ phosphate buffer $(\mathrm{pH}$ 7), containing $0.1 \%(\mathrm{v} / \mathrm{v}$ ) Triton X-100 (pH 7) (for superoxide dismutase and catalase). Cell suspensions were homogenized and centrifuged at $16,000 \times \mathrm{g}$ for $30 \mathrm{~min}$ at $4^{\circ} \mathrm{C}$. The resulting extracts were used for spectrophotometric measurement of enzymatic activity and for the determination of total protein concentration (cat. 500-0006, Bio-Rad Laboratories, Milan, Italy), using BSA as the standard [27]. All spectrophotometric readings were carried out in triplicate by using a Lamba25 spectrophotometer (PerkinElmer Inc., Waltham, MA, USA). With regard to enzymatic activity assessments, within-assay coefficient of variations ranged from $1.44 \%$ to $6.82 \%$, depending on the enzyme analyzed.

\subsection{Total Superoxide Dismutase (tSOD) Enzymatic Activity.} The tSOD (EC 1.15.1.1) activity was assayed by measuring its ability to inhibit the autoxidation of epinephrine (cat. E4375, Sigma-Aldrich, Milan, Italy), which was spectrophotometrically monitored at $480 \mathrm{~nm}$ at $30^{\circ} \mathrm{C}$, according to Sun and Zigman [28]. One unit of tSOD activity was assumed to halve the rate of epinephrine autoxidation. Measurements were carried out in quadruplicate.

\subsection{Catalase (CAT) Enzymatic Activity. The CAT (EC 1.11.1.6)} activity was assayed by monitoring the decomposition of $10 \mathrm{mM}$ hydrogen peroxide at $240 \mathrm{~nm}$ (cat. 21,676-3, SigmaAldrich), as described by Aebi [29]. One unit of enzyme activity was defined as $1 \mu \mathrm{mol}$ of $\mathrm{H}_{2} \mathrm{O}_{2}$ reduced/min at $25^{\circ} \mathrm{C}$. Measurements were carried out in five replicates.
2.5. Total Glutathione Peroxidase (GPX) Enzymatic Activity. The total GPX (EC 1.11.1.9) activity was determined by following the oxidation of nicotinamide adenine dinucleotide phosphate (NADPH) at $340 \mathrm{~nm}$ in a glutathione reductasecoupled reaction, using cumene hydroperoxide as substrate (cat. C0524, Sigma-Aldrich) [30]. One unit of enzyme activity was defined as $1 \mu \mathrm{mol}$ of $\mathrm{GSH}$-conjugated $/ \mathrm{min}$ at $25^{\circ} \mathrm{C}$. Measurements were carried out in quadruplicate.

2.6. Glyoxalase 1 (GLO1) Enzymatic Activity. The GLO1 (EC 4.4.1.5) activity was assayed by recording appearance of (R)-Slactoylglutathione at $240 \mathrm{~nm}$, as described by Mannervik and colleagues [31], in a reaction mixture that contained $1 \mathrm{mM}$ GSH (cat. G4251, Sigma-Aldrich) and $2 \mathrm{mM}$ methylglyoxal (cat. M0252, Sigma-Aldrich). One unit of enzyme activity was defined as $1 \mu \mathrm{mol}$ of (R)-S-lactoylglutathione formed/min at $25^{\circ} \mathrm{C}$. Measurements were carried out in quadruplicate.

\subsection{Glyoxalase 2 (GLO2) Enzymatic Activity and Glutathione} Assay. The GLO2 (EC 3.1.2.6) activity was assayed by recording at $240 \mathrm{~nm}$ the disappearance of $0.3 \mathrm{mM}$ (R)-Slactoylglutathione (cat. L7140, Sigma-Aldrich), as described by Guha and coworkers [32]. One unit of enzyme activity was defined as $1 \mu \mathrm{mol}$ of lactoylglutathione hydrolyzed $/ \mathrm{min}$ at $25^{\circ} \mathrm{C}$. Measurements were carried out in quadruplicate.

Total (tGSH) and oxidized glutathione (GSSG) levels were measured by using the glutathione assay kit, according to the method described by Baker and colleagues [33]. Briefly, $\sim 150 \mathrm{mg}$ of tissue was homogenized in 5 volumes of MES buffer (provided by the kit) and centrifuged at $10,000 \times \mathrm{g}$ for $15 \mathrm{~min}$, at $4^{\circ} \mathrm{C}$. Supernatants were immediately treated with 5\% (w/v) metaphosphoric acid (cat. 239275, Sigma-Aldrich) and centrifuged at $4,000 \times \mathrm{g}$ for $5 \mathrm{~min}$, as recommended by the supplier. $50 \mu \mathrm{L}$ of protein-free supernatants (diluted $1: 3$ in MES buffer) was added to 3 volumes of Assay Cocktail. The color development was followed at $405 \mathrm{~nm}$ for $25 \mathrm{~min}$, through a Victor3 microplate reader (PerkinElmer). Parallel assessments of GSSG were carried out by first derivatizing reduced glutathione with 2-vinylpyridine (cat. 132292, SigmaAldrich), as recommended by the manufacturer. Standardbased calibration curves were obtained from pure GSSG- and GSH-containing reactions (range: 0-8 $\mu \mathrm{M}$ GSSG, $0-16 \mu \mathrm{M}$ tGSH). All samples were assessed in triplicate. Results were shown as GSSG over GSH ratios, with the latter component calculated as total GSH-2GSSG. Within- and between-assay coefficients of variations were equal to $1.6 \%$ and $3.6 \%$, respectively.

2.8. RNA Extraction and Real-Time RT-PCR. RNA was extracted from ovaries by using the Applied Biosystems ${ }^{\circledR}$ Picopure kit (cat. 12204-01, Thermo Fisher Scientific, Inc., Waltham, MA, USA), and the contaminant DNA was degraded by Invitrogen ${ }^{\mathrm{TM}}$ DNA-free kit (cat. AM1906, Thermo Fisher Scientific, Inc.), following the supplier's recommendations. The resulting RNA was used to obtain cDNA via reverse transcription (Life Technologies SuperScript VILO cDNA Synth Kit, cat. 11754050, Thermo Fisher Scientific, Inc.). 
TABle 1: Primer pairs used for amplicon generation.

\begin{tabular}{lccc}
\hline & Forward $\left(5^{\prime} \rightarrow 3^{\prime}\right)$ & Reverse $\left(5^{\prime} \rightarrow 3^{\prime}\right)$ & Reference \\
\hline GLO1 & GATTTGGTCACATTGGGATTGC & TTCTTTCATTTTCCCGTCATCAG & {$[34]$} \\
GLO2 & GGGAACGAGAAGCTGGTGAA & CCGAAGTATGGCAGGGTGTT & 1 \\
$\beta$-Actin & GAGACCTTCAACACCCCAGC & ATGTCACGCACGATTTCCC & 2 \\
\hline
\end{tabular}

${ }^{2}$ Primer blast (accession number NM_007393.5).

${ }^{1}$ Primer blast (accession number NM_024284.2).

The cDNA was used (dil. 1:50) for the PCR amplification which was performed in an Applied Biosystems 7300 system (Thermo Fisher Scientific, Inc.) by using the PowerSYBR Green PCR Master Mix kit (cat. 4367659, Thermo Fisher Scientific, Inc.). Applied Biosystems custom oligos were purchased from Thermo Fisher Scientific, Inc. (Table 1).

Amplification steps were set as follows: initial denaturation at $95^{\circ} \mathrm{C}$ for $2 \mathrm{~min}$ and 40 cycles of $95^{\circ} \mathrm{C}$ for 5 seconds and $60^{\circ} \mathrm{C}$ for 30 seconds. In order to verify coamplification of unspecific targets, melting curves were performed for all primer pairs $\left(95^{\circ} \mathrm{C}\right.$ for 15 seconds, $60^{\circ} \mathrm{C}$ for $1 \mathrm{~min}, 95^{\circ} \mathrm{C}$ for 15 seconds, and $60^{\circ} \mathrm{C}$ for 15 seconds). Gene expression was calculated by using the $\Delta \Delta \mathrm{Ct}$ method, with $\beta$-actin used as the reference transcript for the relative quantitation and S2 considered as the calibrator sample (100\% gene expression) [35]. All samples were processed in six replicates. Withinassay coefficient of variation ranged from $0.94 \%$ and $1.24 \%$, depending on the transcript analyzed.

2.9. Western Immunoblot. Ovaries were lysed $(300 \mathrm{mg} / \mathrm{mL})$ in RIPA buffer (cat. R0278, Sigma-Aldrich) that was supplemented with protease inhibitors (cat. P8340, Sigma-Aldrich) and phosphatase inhibitors (cat. P2850 and P5726, SigmaAldrich). After centrifugation at $16,000 \times \mathrm{g}$ for $30 \mathrm{~min}$ at $4^{\circ} \mathrm{C}$, supernatants were assayed for total protein content, by using the BCA Protein Assay Kit and bovine serum albumin as the standard (cat. 23225, Thermo Fisher Scientific, Inc.). Denatured samples were run in triplicate on $12 \%$ polyacrylamide gels according to Laemmli [36]. Protein bands were transferred onto polyvinylidene difluoride (PVDF) membranes by electrophoretic transfer [37]. Nonspecific binding sites were blocked at room temperature for one hour with $5 \%(\mathrm{w} / \mathrm{v})$ Blotting-Grade Blocker (cat. 170-6404, Bio-Rad Laboratories), in Tris-buffer saline containing $0.05 \%$ (v/v) Tween20 (cat. P5927, Sigma-Aldrich) (TBS-T). Membranes were incubated overnight with TBS-T-diluted primary antibodies. PVDF sheets were incubated with peroxidase-conjugated secondary antibodies for two hours (see Section 2.11 for details about dilutions). The specific immune complexes were detected by using the Metal Enhanced DAB Substrate Kit (cat. 34065), as recommended by the supplier (Thermo Fisher Scientific, Inc.). Digital images were processed through the TotalLab software (TotalLab Ltd., Newcastle upon Tyne, UK). Signal normalization was carried out by using $\beta$-actin as the loading control protein, and results were given as arbitrary units. Results for argpyrimidine levels were obtained by analyzing one band found exclusively in blots incubated with both antibodies, by subtracting nonspecific signals obtained by omitting the primary antibody. Experiments were performed in triplicate. Within-assay coefficient of variation ranged from $0.20 \%$ to $6.31 \%$, depending on the protein analyzed.

2.10. Protein Carbonyl Content Assessment. The reaction between protein carbonyls and 2,4-dinitrophenylhydrazine (DNPH) was used to detect the carbonyl content in our samples [38]. Briefly, ovaries $(\sim 150 \mathrm{mg})$ were rinsed with phosphate buffered saline solution and homogenized in $500 \mu \mathrm{L}$ of cold EDTA-containing phosphate buffer ( $\mathrm{pH}$ 6.7). Samples were centrifuged at $10,000 \times \mathrm{g}$ for 15 minutes at $4^{\circ} \mathrm{C}$. In order to remove contaminant nucleic acids, supernatants were treated with $1 \%(\mathrm{w} / \mathrm{v})$ streptomycin sulfate (cat. sc202821, Santa Cruz Biotechnology, Inc., Santa Cruz, CA, USA), as recommended by the manufacturer. After reacting with DNPH, samples were transferred into 96-well plate, and the formation of the corresponding hydrazones was followed by photometric reading (Victor ${ }^{3}$, PerkinElmer Inc.) at $370 \mathrm{~nm}$ $\left(0.022 \mu \mathrm{M}^{-1} * \mathrm{~cm}^{-1}\right)$. Results were normalized against proteins that were still present in the wells at the moment of the reading, as suggested by the manufacturer. Experiments were performed in triplicate. Within- and between-assay coefficients of variations were equal to $4.7 \%$ and $8.5 \%$, respectively.

2.11. Antibodies. The anti-AMH (cat. ab103233) (dil. 1:50) and anti- $\beta$-actin (cat. ab8227) (dil. 1:6000) antibodies were supplied by Abcam (Cambridge, UK). The anti-argpyrimidine antibody (cat. NOF-N213430-EX) (dil. 1:75) was provided by Cosmo Bio Co., Ltd. (Tokyo, Japan). The peroxidaseconjugated anti-mouse secondary antibody (cat. A9044) (dil. $1: 1000$ ) was purchased from Sigma-Aldrich. The peroxidaseconjugated anti-rabbit secondary antibody (cat. PI1000) (dil. $1: 1000$ ) was supplied by DBA Italia (Milan, Italy). Protein Carbonyl colorimetric assay kit (cat. 10005020) and Glutathione Assay Kit (cat. 703002) were purchased from Cayman Chemical Co. (Ann Arbor, MI, USA).

2.12. Statistics. Factorial (exercise $\times$ time) ANOVA and Holm-Sidak post hoc tests for multiple comparisons were used for statistical analysis (Statsoft Statistica 10 and SyStat SigmaStat v3.5). The null hypothesis was rejected with $P<$ 0.05. Real-time PCR results were reported as means \pm error estimation of the calculated ratio using the Taylor's series, as appropriate for the $\Delta \Delta \mathrm{Ct}$ method [39]. All other results were given as means \pm standard deviations. 


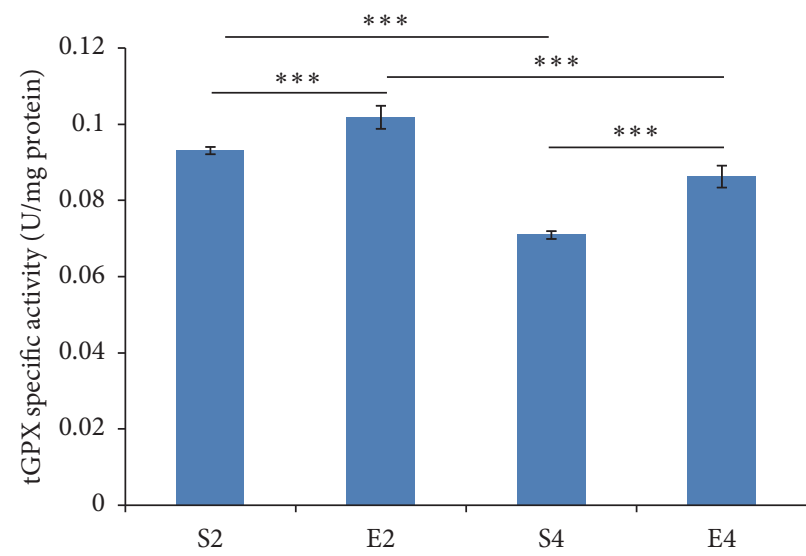

(a)

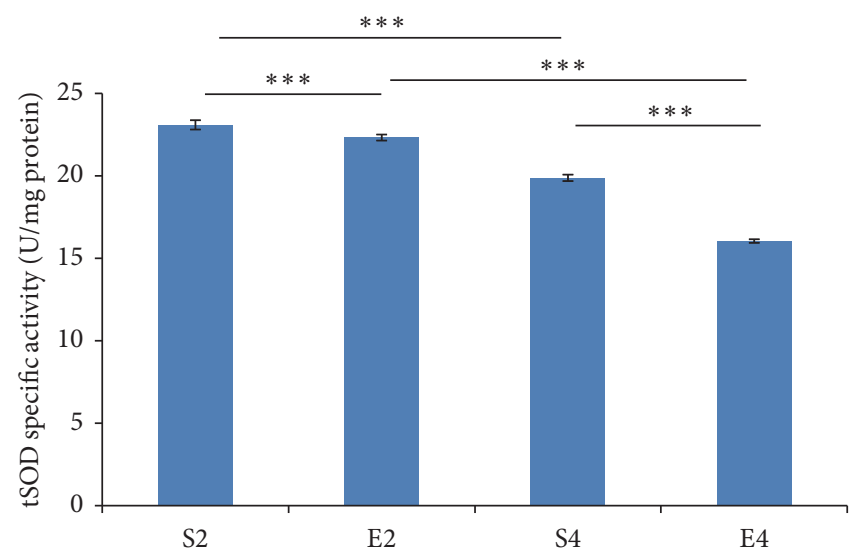

(b)

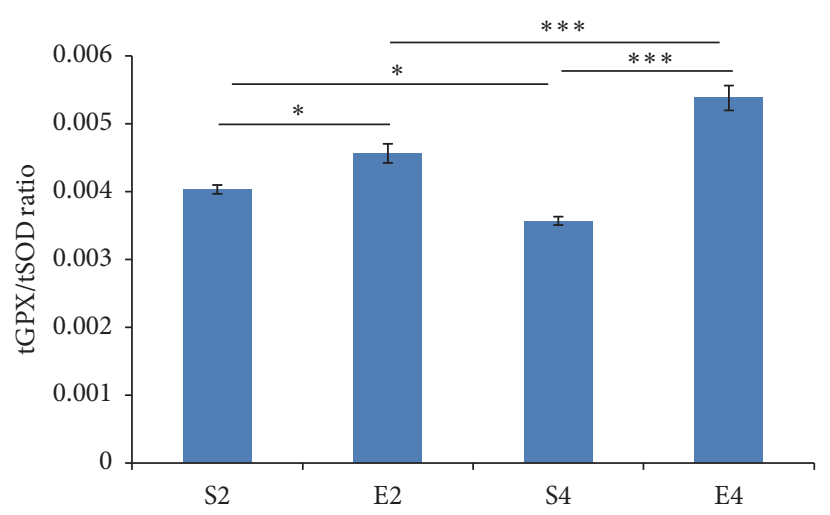

(c)

FIGURE 2: Ovarian total glutathione peroxidase (tGPX)/total superoxide dismutase (tSOD) antioxidant capacities in mice after regular and moderate treadmill running. Specific activities ( $a$ and b) and efficiency of the tGPX/tSOD enzymatic coupling (c) in ovaries of middle-aged $\mathrm{CD} 1$ female mice after two- or four-month running programme (E2 or E4, resp.); age-matched sedentary animals (S2, S4) were used as controls ( $n=12$, each group). Values were given as means \pm std. dev. and the level of statistical significance was calculated by using factorial $(2 \times 2)$ ANOVA and Holm-Sidak post hoc tests for multiple comparisons: ${ }^{*} P<0.05$; ${ }^{* * *} P<0.001$.

\section{Results}

3.1. Effects of Age and Exercise on Ponderal State and Food Intake. In coherence with our previous results [16, 17], no effect of age or running was observed on either ponderal state or food consumption (not shown).

\subsection{Effect of Exercise on ROS-Targeting Enzymatic Defence}

3.2.1. Total Glutathione Peroxidase ( $t G P X)$. We found significant main effects of both age and exercise on the total glutathione peroxidase specific activity $(P<0.001$, Cohen's $d$-based effect sizes $=0.979$ and 0.939 , resp.). In addition, we found a significant interaction effect $(P<0.01)$. The post hoc test revealed that the transition from 12 to 14 months of age caused a marked reduction of tGPX specific activity in the ovarian environment of sedentary mice $(P<0.001$, Cohen's $d$-based effect size $=0.996$, S4 versus S2), whereas the tGPX activity was significantly increased by both twoand four-month running $(P<0.001$, Cohen's $d$-based effect sizes $=0.890$ and 0.963 , resp. $)(E 2$ versus S2 and E4 versus S4; Figure 2(a)).
3.2.2. Total Superoxide Dismutase (tSOD). Significant main effects of both age and exercise on the total SOD specific activity were found $(P<0.001$ and $P<0.001$, Cohen's $d$ based effect sizes $=0.997$ and 0.986 , resp.). The multiple comparison analysis showed that the tSOD specific activity was significantly reduced by the transition from 12 to 14 months in the ovaries of sedentary rodents $(P<0.001$, Cohen's $d$-based effect size $=0.989)$ (S4 versus S2; Figure 2(b)). Moreover, the age-dependent decline of tSOD activity was greater in exercised mice $(P<0.001$, Cohen's $d$-based effect sizes $=$ 0.851 and 0.997, resp.) (E2 versus S2 and E4 versus S4, Figure 2(b)). Accordingly, we found a significant interaction effect $(P<0.001)$.

3.2.3. $t G P X / t S O D$ Ratio. The $\mathrm{tGPX} / \mathrm{tSOD}$ ratios were also examined, as such a proportion is better indicative of the efficiency of the antioxidative enzymatic defence than each activity alone [40, 41]. Significant main effects of both age and moderate running on the tGPX/tSOD ratio were found $(P<0.05$ and $P<0.001$, Cohen's $d$-based effect sizes $=$ 0.614 and 0.979 , resp.). In addition, we found a significant interaction effect $(P<0.001)$. The post hoc test showed that 


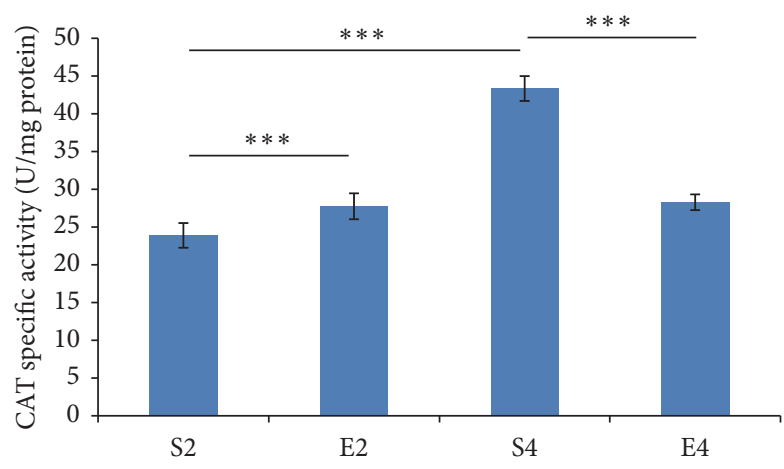

(a)

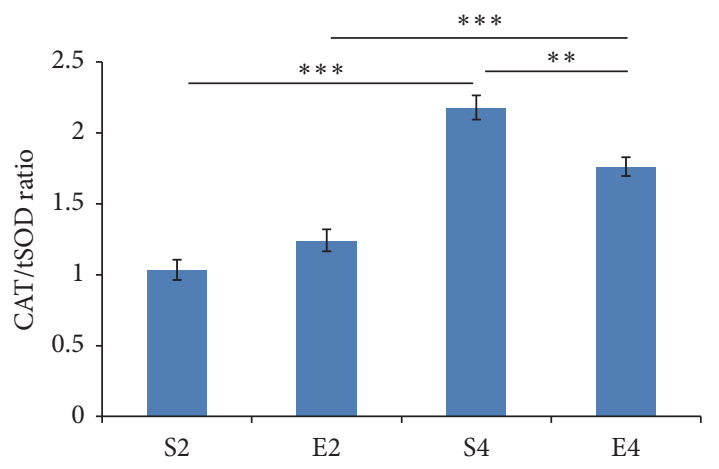

(b)

FIGURE 3: Ovarian catalase (CAT)/total superoxide dismutase (tSOD) antioxidant capacities in mice after regular and moderate treadmill running. CAT specific activity (a) and efficiency (b) of the CAT/tSOD enzymatic coupling in ovaries of middle-aged CD1 female mice after two- or four-month running programme (E2 or E4, resp.); age-matched sedentary animals (S2, S4) were used as controls ( $n=12$, each group). Values were given as means \pm std. dev. and the level of statistical significance was calculated by using factorial $(2 \times 2)$ ANOVA and Holm-Sidak post hoc tests for multiple comparisons: ${ }^{* *} P<0.01 ;{ }^{* * *} P<0.001$.

the ratio between $\mathrm{tGPX/tSOD}$ was significantly reduced by the transition from 12 to 14 months in the ovaries of sedentary animals $(P<0.05$, Cohen's $d$-based effect size $=0.965)$ (S4 versus S2, Figure 2(c)). Interestingly, the age-dependent decline of the tGPX/tSOD ratio was completely reverted by the exercise programme (Figure 2(c)). Moreover, we observed that exercised mice showed increased tGPX/tSOD ratios, after both two- and four-month running $(P<0.05$ and $P<0.001$, Cohen's $d$-based effect sizes $=0.925$ and 0.989 , resp.) (E2 versus S2 and E4 versus S4, Figure 2(c)).

3.2.4. Catalase (CAT). We observed significant main effects of both age and treadmill running on the CAT specific activity $(P<0.001$ and $P<0.001$, Cohen's $d$-based effect sizes $=0.957$ and 0.880 , resp.). As shown in Figure 3(a), the transition from 12 to 14 months of age caused an increase of the CAT specific activity in the ovaries of sedentary mice $(P<0.001$, Cohen's $d$-based effect size $=0.986)(S 4$ versus S2). Interestingly, we did not find any age-dependent change of the CAT specific activity in the ovaries of exercised mice (Figure 3(a)). Accordingly, a significant interaction effect was also found $(P<0.001)$. The CAT specific activity resulted to be elevated by the two-month running regimen $(P<$ 0.001 , Cohen's $d$-based effect size $=0.755$ ), whereas a strong reduction of the CAT activity was observed after the fourmonth running $(P<0.001$, Cohen's $d$-based effect size $=$ 0.984) (E2 versus S2 and E4 versus S4, Figure 3(a)).

3.2.5. CAT/tSOD Ratio. As done for the tGPX and tSOD activities, CAT/tSOD ratios were also considered to better assess the efficiency of the ROS-targeting enzymatic protection. We detected significant main effects of both age and exercise on the CAT/tSOD ratio $(P<0.001$ and $P<0.05$, Cohen's $d$-based effect sizes $=0.984$ and 0.571 , resp.). The ratio between CAT/tSOD was significantly increased by the transition from 12 to 14 months in the ovaries of unexercised mice $(P<0.001$, Cohen's $d$-based effect size $=0.991)$; however we observed that exercised mice showed a strongly reduced CAT/tSOD ratio after four-month running $(P<$ 0.01 , Cohen's $d$-based effect size $=0.939)(S 4$ versus S2 and E4 versus S4, resp., Figure 3(b)). Conversely, the twomonth exercise programme did not change significantly the CAT/tSOD ratio (E2 versus S2, Figure 3(b)). As a result, a significant interaction effect was found because of the fact that the age-dependent elevation of CAT/tSOD ratio was greater in sedentary mice than in exercised rodents $(P<$ $0.001)$.

3.2.6. GSSG/GSH Ratio. Significant main effects of both age and moderate running on the redox balance between GSH and oxidized glutathione were found $(P<0.001$ and $P<$ 0.001 , Cohen's $d$-based effect sizes $=0.937$ and 0.954 , resp.). The GSSG/GSH ratio was not significantly affected by the transition from 12 to 14 months in the ovaries of unexercised mice (S4 versus S2, Figure 4), whereas exercise reduced the GSSG/GSH redox balance after both two- and four-month running $(P<0.05$ and $P<0.001$, Cohen's $d$-based effect sizes $=0.741$ and 0.976 , resp.) (E2 versus S2 and E4 versus S4, Figure 4). Accordingly, a significant interaction effect was found $(P<0.001)$.

3.2.7. Protein Carbonyl Content. Significant main effects of both running and age on the protein carbonyl content were found $(P<0.01$ and $P<0.05$, Cohen's $d$-based effect sizes $=$ 0.931 and 0.787 , resp.). The level of oxidatively modified proteins was not significantly increased by the transition from 12 to 14 months in the ovaries of unexercised mice (Figure 5). Conversely, we found that exercised mice showed a strongly reduced protein carbonyl content after both twoand four-month running $(P<0.05$ and $P<0.001$, Cohen's $d$-based effect sizes $=0.906$ and 0.938 , resp.) (E2 versus $\mathrm{S} 2$ and $\mathrm{E} 4$ versus S4, Figure 5). Accordingly, a near significant interaction effect was also found $(P=0.057)$. 


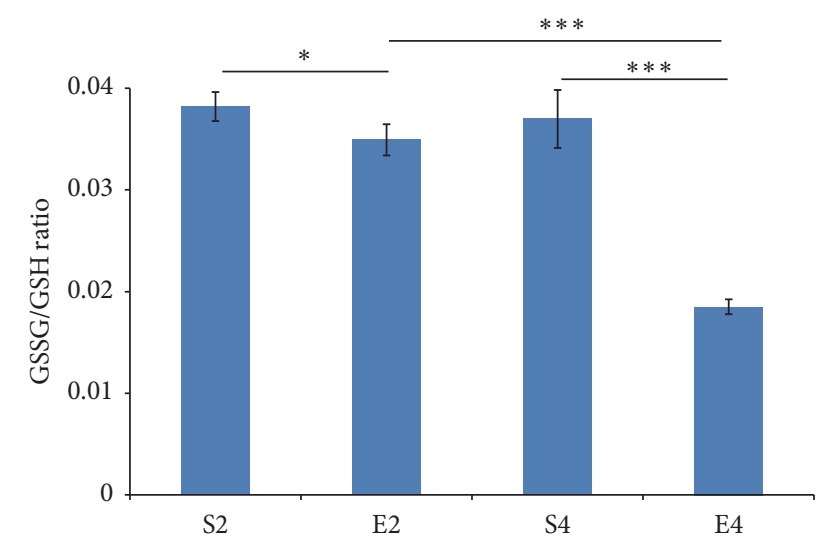

FIgURE 4: Ovarian balance of glutathione redox couple in mice after regular and moderate treadmill running. Glutathione disulfide (GSSG) over glutathione (GSH) ratio in ovaries of middle-aged CD1 female mice after two- or four-month running programme (E2 or $\mathrm{E} 4$, resp.); age-matched sedentary animals (S2, S4) were used as controls ( $n=12$, each group). Values were given as means \pm std. dev. and the level of statistical significance was calculated by using factorial $(2 \times 2)$ ANOVA and Holm-Sidak post hoc tests for multiple comparisons: ${ }^{*} P<0.05 ;{ }^{* * *} P<0.001$.

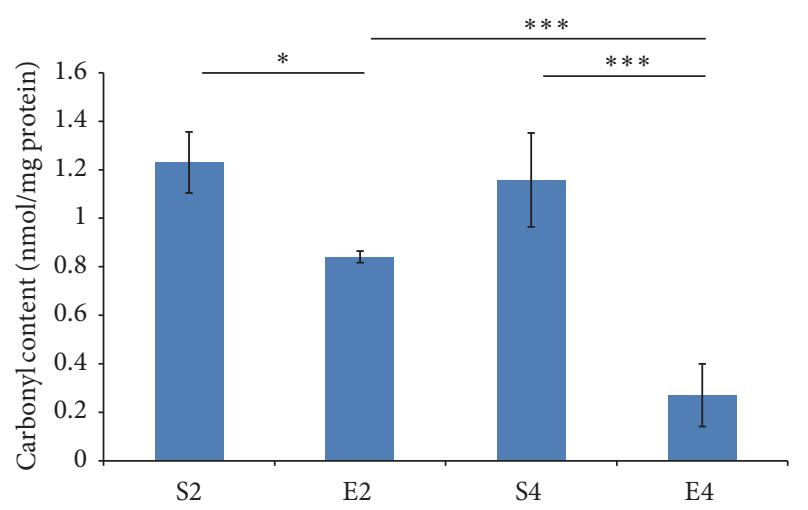

FIGURE 5: Ovarian levels of oxidatively modified proteins in mice after regular and moderate treadmill running. Protein carbonyl content in ovaries of middle-aged CD1 female mice after two- or four-month running programme (E2 or E4, resp.); age-matched sedentary animals (S2, S4) were used as controls $(n=12$, each group). Values were given as means \pm std. dev. and the level of statistical significance was calculated by using factorial $(2 \times 2)$ ANOVA and Holm-Sidak post hoc tests for multiple comparisons: ${ }^{*} P<0.05 ;{ }^{* * *} P<0.001$.

\subsection{Effect of Exercise on MG-Related Metabolism}

3.3.1. Glyoxalase 1 (GLO1) Expression and Activity. We observed a significant main effect of age on the level of GLO1 mRNA $(P<0.001$, Cohen's $d$-based effect size $=0.950)$. As shown in Figure 6(a), the transition from 12 to 14 months of age increased GLO1 mRNA level in the ovarian environment of sedentary mice $(P<0.001$, Cohen's $d$-based effect size $=$ 0.968) (S4 versus S2). The age-related increase of GLO1
mRNA level was strongly limited in exercised animals; therefore a significant interaction effect was also found $(P<$ 0.001). Accordingly, the GLO1 transcript was found to be significantly reduced by the four-month exercise, whereas an increase in GLO1 mRNA amount after two-month running was observed $(P<0.001$, Cohen's $d$-based effect sizes $=0.854$ and 0.876) (E4 versus S4 and E2 versus S2) (Figure 6(a)).

We detected a significant main effect of age on the specific activity of GLO1 $(P<0.001$, Cohen's $d$-based effect size $=$ 0.785). A near significant main effect of moderate running on the GLO1 specific activity was also found $(P=0.07$, Cohen's $d$-based effect size $=0.451$ ). As shown in Figure 6(b), in unexercised mice the GLO1 specific activity within the ovaries resulted to be lowered by the passage from 12 to 14 months of age $(P<0.001$, Cohen's $d$-based effect size $=0.981)$ (S4 versus S2). Conversely, in the ovaries of exercised mice the GLO1 specific activity was increased in an age-dependent fashion $(P<0.001$, Cohen's $d$-based effect size $=0.834)(\mathrm{E} 4$ versus E2) (Figure 6(b)). Accordingly, a significant interaction effect was found $(P<0.001)$. In fact, the GLO1 specific activity was reduced by the two-month running regimen and increased by the four-month exercise programme $(P<0.001$, Cohen's $d$-based effect sizes $=0.877$ and 0.854 , resp.) $(\mathrm{E} 2$ versus S2 and E4 versus S4, Figure 6(b)).

3.3.2. Glyoxalase 2 (GLO2) Expression and Activity. We found significant main effects of both age and physical exercise on GLO2 mRNA amount $(P<0.001$ and $P<0.001$, Cohen's $d$-based effect sizes $=0.751$ and 0.702 , resp.). As shown in Figure 7(a), the transition from 12 to 14 months of age caused a marked decrease of GLO2 mRNA level in the ovaries of sedentary rodents $(P<0.001$, Cohen's $d$-based effect size $=$ 0.838 ) (S4 versus S2). On the contrary, exercise completely reverted the age-dependent decline of the GLO2 mRNA level $(P<0.001$, Cohen's $d$-based effect size $=0.990)(\mathrm{E} 4$ versus E2) (Figure 7(a)). Accordingly, a significant interaction effect was also found $(P<0.001)$. In fact, the amount of GLO2 transcript was reduced by the two-month exercise, whereas a higher GLO2 mRNA amount was found in the ovaries of mice that underwent the four-month running programme $(P<0.001$, Cohen's $d$-based effect sizes $=0.959$ and 0.952 , resp.) (E2 versus S2 and E4 versus S4, Figure 7(a)).

In addition, we observed a significant main effect of age on GLO2 specific activity $(P<0.001$, Cohen's $d$-based effect size $=0.916$ ). The specific activity of GLO2 within the ovary was reduced by the transition from 12 to 14 months of age in both sedentary and exercised rodents $(P<0.001$, Cohen's $d$-based effect sizes $=0.958$ and 0.874$)(\mathrm{S} 4$ versus S2 and $\mathrm{E} 4$ versus E2, Figure 7(b)). Accordingly, exercise did not affect the GLO2 specific activity, after either two- or four-month running (Figure 7(b)).

3.3.3. Argpyrimidine. We detected main effects of both age and treadmill running on the amount of MG-dependent protein damage $(P<0.001$ and $P<0.01$, Cohen's $d$ based effect sizes $=0.969$ and 0.836 , resp.). As reported in Figure 8, we found that the levels of ovarian argpyrimidine increased in an age-dependent fashion in both sedentary 


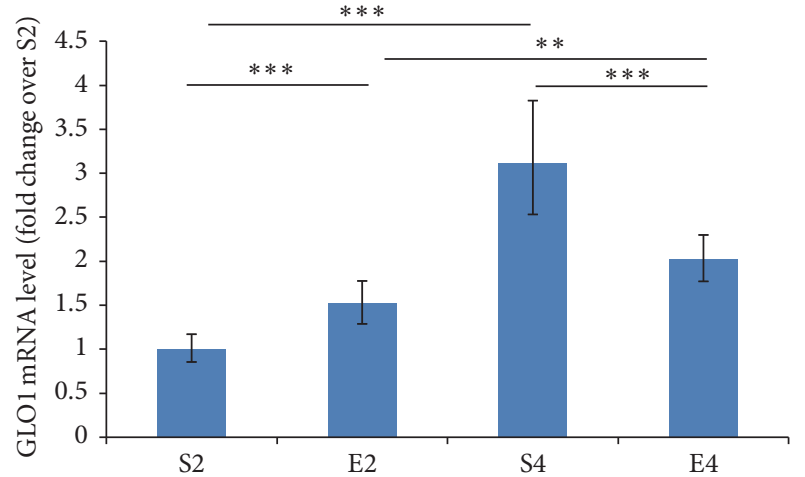

(a)

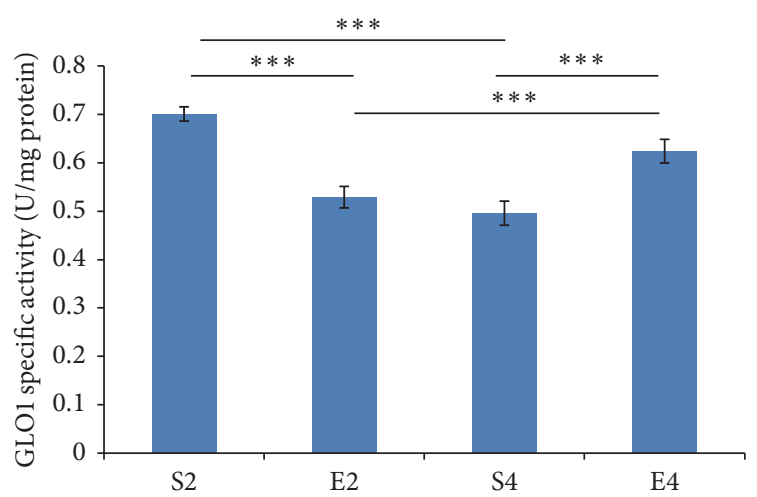

(b)

FIGURE 6: Ovarian transcriptional expression and enzymatic activity of glyoxalase 1 (GLO1) in mice after regular and moderate treadmill running. GLO1 mRNA levels (a) and specific activity (b) of GLO1 in ovaries of middle-aged CD1 female mice after two- or four-month running programme (E2 or E4, resp.); age-matched sedentary animals (S2, S4) were used as controls $(n=12$, each group). Real-time PCR results were reported as means \pm error estimation of the calculated ratio using Taylor's series, as appropriate for the DDCt method [39]. Specific activities values were given as means \pm std. dev. The level of statistical significance was calculated by using factorial $(2 \times 2)$ ANOVA and Holm-Sidak post hoc tests for multiple comparisons: ${ }^{* *} P<0.01 ;{ }^{* * *} P<0.001$.

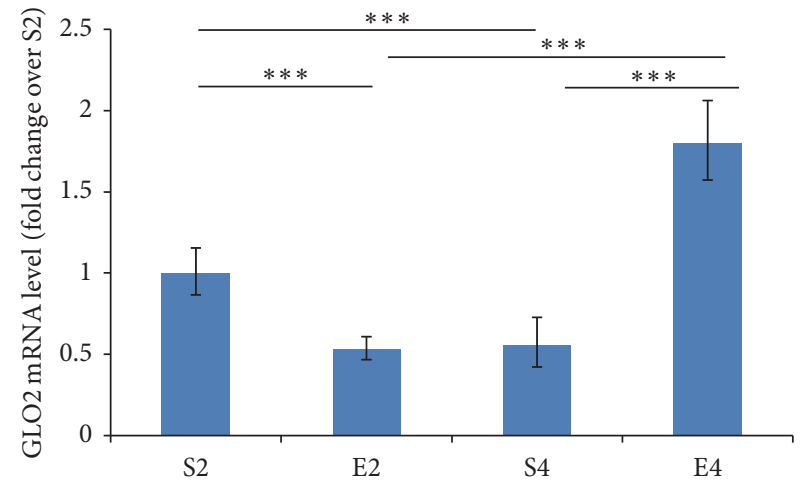

(a)

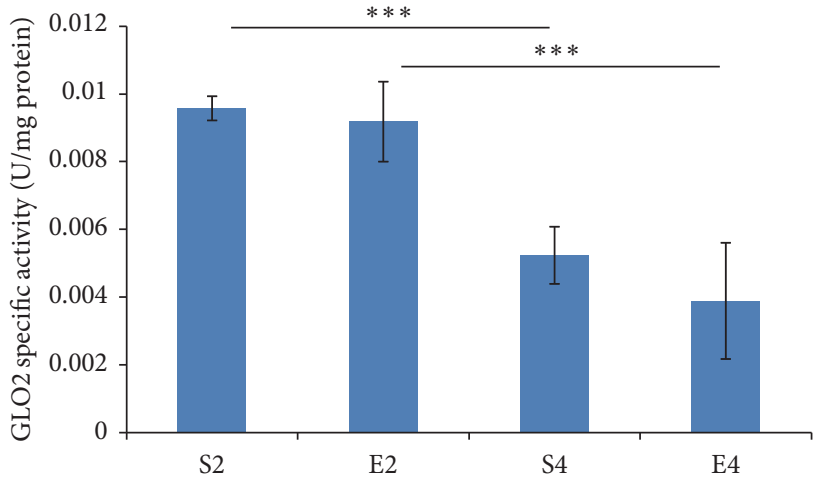

(b)

FIGURE 7: Ovarian transcriptional expression and enzymatic activity of glyoxalase 2 (GLO2) in mice after regular and moderate treadmill running. GLO2 mRNA levels (a) and specific activity (b) of GLO2 in ovaries of middle-aged CD1 female mice after two- or four-month running programme (E2 or E4, resp.); age-matched sedentary animals (S2, S4) were used as controls $(n=12$, each group). Real-time PCR results were reported as means \pm error estimation of the calculated ratio using Taylor's series, as appropriate for the DDCt method [39]. Specific activities values were given as means \pm std. dev. The level of statistical significance was calculated by using factorial $(2 \times 2)$ ANOVA and Holm-Sidak post hoc tests for multiple comparisons: ${ }^{* * *} P<0.001$.

and exercised mice $(P<0.01$, Cohen's $d$-based effect sizes $=0.867$ and 0.978, resp.) (S4 versus S2 and E4 versus E2) (Figure 8). Interestingly, the levels of argpyrimidine resulted to be decreased after two-month running $(P<0.05$, Cohen's $d$-based effect size $=0.877)$, whereas the four-month exercise regimen increased the amount of the major MG-related protein adduct $(P<0.001$, Cohen's $d$-based effect size $=$ 0.936) (E2 versus S2 and E4 versus S4, resp., Figure 8). A significant interaction effect was also found $(P<0.001)$.

3.4. Effect of Exercise on Ovarian Reserve. A significant main effect of age on the AMH level was found $(P<0.001$, Cohen's $d$-based effect size $=0.997)$. In addition, we detected a significant interaction effect $(P=0.01)$. The passage from 12 to 14 months of age reduced the protein levels of the ovarian reserve marker AMH in both sedentary and exercised mice
$(P<0.001$, Cohen's $d$-based effect sizes $=0.995$ and 0.992, resp.) (S4 versus S2 and E4 versus E2, Figure 9). However, the four-month running increased significantly AMH levels $(P<0.05$, Cohen's $d$-based effect sizes $=0.769)($ E4 versus S4), whereas no significant effect was found after two months of running (Figure 9).

\section{Discussion}

One of the major epidemiologic trends in modern Western societies is the rise of chronic and degenerative diseases associated with the unprecedented rate of population aging $[42,43]$. For this reason, noninvasive and inexpensive interventions aimed at ensuring healthy aging are becoming one of the most urgent needs in biomedical research. 


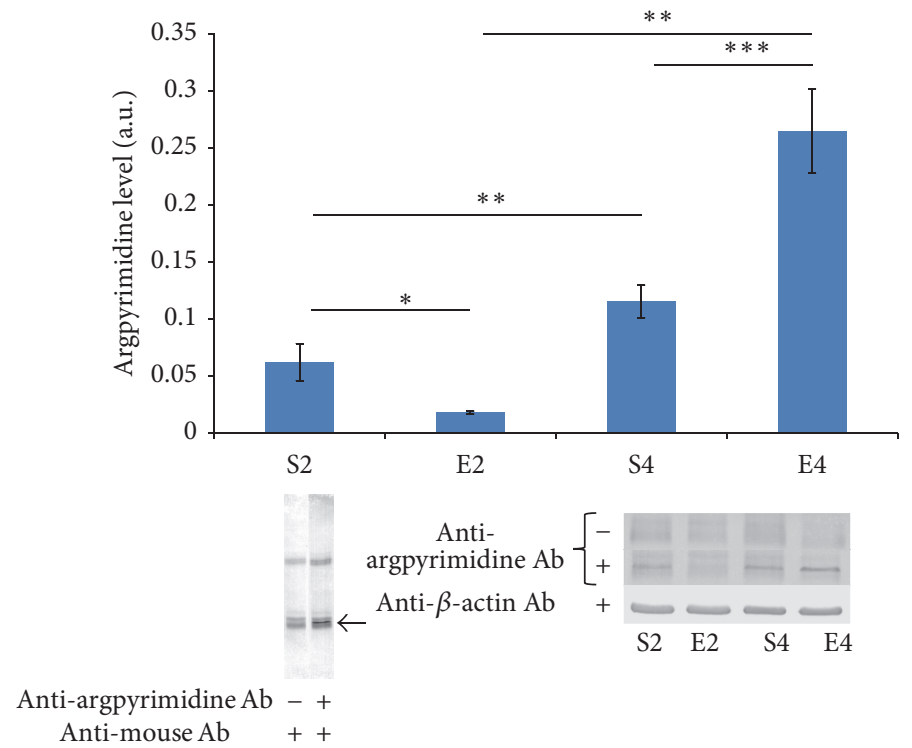

FIGURE 8: Ovarian methylglyoxal-dependent protein damage in mice after regular and moderate treadmill running. Argpyrimidine-directed Western immunoblot in ovaries of middle-aged CD1 female mice after two- or four-month running programme (E2 or E4, resp.); age-matched sedentary animals (S2, S4) were used as controls $(n=12$, each group). Immunosignals were normalized against the housekeeping protein $\beta$ actin. Measurements were carried out by analyzing the single band (arrow) that was found exclusively in blots incubated with both antibodies, by subtracting nonspecific signals obtained by omitting the primary antibody (bottom-left corner). Representative Western blots of four independent experiments were reported (bottom-right corner). Values were given as means \pm std. dev. and the level of statistical significance was calculated by using factorial $(2 \times 2)$ ANOVA and Holm-Sidak post hoc tests for multiple comparisons: ${ }^{*} P<0.05 ;{ }^{* *} P<0.01 ;{ }^{* * *} P<0.001$.

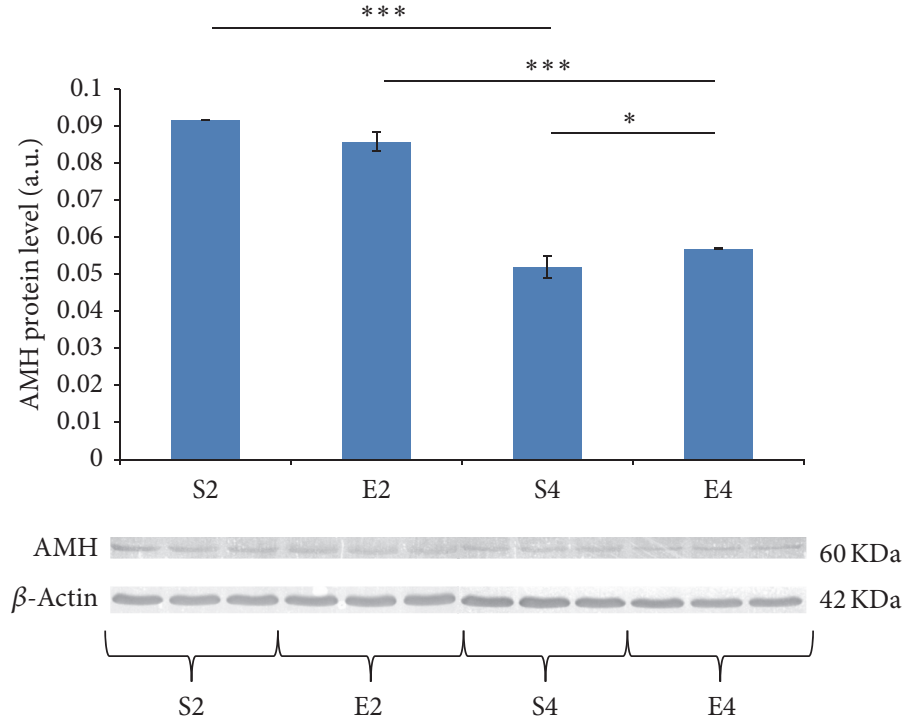

FIGURE 9: Ovarian levels of anti-Müllerian hormone (AMH) in mice after regular and moderate treadmill running. AMH-directed Western immunoblot in ovaries of middle-aged CD1 female mice after two- or four-month running programme (E2 or E4, resp.); age-matched sedentary animals (S2, S4) were used as controls ( $n=12$, each group). Immunosignals were normalized against the housekeeping protein $\beta$-actin. Representative Western blots of four independent experiments were reported. Values were given as means \pm std. dev. and the level of statistical significance was calculated by using factorial $(2 \times 2)$ ANOVA and Holm-Sidak post hoc tests for multiple comparisons: ${ }^{*} P<0.05$; ${ }^{* * *} P<0.001$. 
We already showed that long-term moderate running in the middle age elicits antiaging effects in the brain of CD1 mice, mainly by activating the major scavenging systems against ROS and MG $[16,17]$, which are both believed to be key factors involved in ovarian aging [9-11]. In mice mean reproductive lifespan is 30 months during which increasing irregularity in ovulatory cycles appears at approximately 10 months, followed by exhaustion of oocytes at 24 months [44]. In order to establish whether and how exercise may delay the progression of the age-related biomolecular burden, we chose the biological period ranging from 10 to 14 months of rodent age. The levels of anti-Müllerian hormone (AMH), which is a widely used marker of ovarian reserve [21, 22], resulted to be dramatically reduced during this period, thus mirroring the physiological decline of primordial follicle pool [19]. The tGPX/tSOD ratio, which is a reliable indicator of the cellular scavenging efficiency against the $\mathrm{O}_{2}{ }^{--} / \mathrm{H}_{2} \mathrm{O}_{2}$ redox couple $[7,17,45]$, also declined in an age-dependent fashion. Since the mammalian GPX acts as the very first line of defence against hydrogen peroxide in vivo, exhibiting greater affinity for $\mathrm{H}_{2} \mathrm{O}_{2}$ than CAT [46], such an early reduction of the GPX-based antioxidant efficiency could lead to the decreased antioxidant protection observed in aged mice $[8,47,48]$. Interestingly, an age-dependent increase in the level of protein oxidation was not found maybe due simultaneous increase of the CAT/tSOD ratio. Given its extremely high turnover rate [49], CAT may be upregulated to compensate the reduced GPX-mediated protection against hydrogen peroxide, thus explaining why both GSH pool and oxidative damage level were preserved.

Our findings confirmed that the increased susceptibility to MG-related damage may play a role in ovarian aging [11]. In fact, the activities of both GLO1 and GLO2 were reduced in an age-dependent fashion. We also found that the impaired defence against MG in the early-aging mouse ovary triggered an attempt of molecular adaptive response through the upregulation of GLO1 gene. Interestingly, the ovarian transcriptional response failed to prevent the agedependent accumulation of MG-related protein damage.

In summary, we identified a biological window (from 12 to 14 months of age) in which a decline of the ovarian reserve occurs along with a strong weakening of both antioxidative and antiglycative protection, and such period was used as an early ovarian aging model over which a moderate exercise programme could be tested for possible antiaging effects.

To the best of our knowledge, no study has ever investigated the role played by physical exercise in regulating the biochemical defence against oxidative and dicarbonyl stress in the ovarian environment during the transition from adult to middle age; hence the comparison between our findings and the available literature was not an easy task. In the present research, mice were subjected to a regular running protocol initiated at 10 months of age, that is, before the biological window in which biochemical and molecular changes occur.

The exercise programme elicited a major effect on the efficiency of the major ROS scavenging enzymes. The running regimen reverted the age-dependent drop of the GPX-based protection and limited the compensatory growth of the CATbased antioxidative defence. More importantly, we observed that the running protocol lowered the amount of oxidatively modified proteins, after both 2 and 4 months of running. Interestingly, the effects of running on the redox balance of glutathione were superimposable with those induced by exercise on the levels of protein carbonyls, especially after 4 months of running. Given the critical role of glutathione in the overall antioxidant protection as the major thioldisulphide redox buffer of the cell [50], it may be conceived that the improvement of the oxidative profile in the ovaries of exercising rodents could be linked to the elevation of available GSH equivalents that was triggered by long-term running. Others have shown that rodents undergoing moderateintensity exercise for 12 weeks exhibit reduced oxidative stress and increased GSH/GSSG ratio in aging aortas [51]. However, we here provide the first evidence of a significant protective effect of habitual running on major redox- and oxidative stress-related parameters within the ovaries of reproductively aging mice. This suggests that moderate running, even when initiated lately in life in mammals that are unfamiliar with exercise, may still be able to improve the redox milieu of the major regulator of female fertility. Our findings may gain importance as some researchers have recently shown that mice harboring gene mutations that promote chronic oxidative stress show accelerated ovarian aging [12]. Accordingly, other authors have demonstrated that the enrichment of the antioxidant defence against ROS is able to postpone the process of aging in mouse ovaries and oocytes [52, 53]. In addition, the treatment with $\mathrm{N}$-acetylcysteine (NAC), a potent low-molecular weight intracellular antioxidant and precursor of GSH, has been shown to improve the follicular function and development by preventing oxidative stress [54].

Oxidative stress and dicarbonyl overload are tightly interlinked phenomena $[5,11]$. Here we show that the enzymatic detoxification of methylglyoxal, as well as the MG-derived protein damage profile, was also affected by the exercise programme. In fact, the age-dependent decline of the specific activity of GLO1 was almost completely reverted by the four-month running treatment. In addition, the age-related upregulation of the GLO1 mRNA was almost totally abolished by the four-month running regimen, thus suggesting that the long-term exercise protocol abolished the age-driven perturbation of the GLO1 expression. However, the longterm exercise regimen sharpened the age-related drop of the enzymatic activity of GLO2, yet GLO2 mRNA was strongly increased by the chronic running programme. Since GLO2 is the enzyme that catalyzes the rate-limiting step of MG removal $[55,56]$, we were not surprised to observe that the four-month running regimen exacerbated the MGdependent carbonyl stress despite the apparent activation of the GLO1-based protection. This may suggest that long-term running could be able to enhance the endogenous formation of $\mathrm{MG}$ or reduce the activity of the physiological removal mechanisms of glycated proteins. In this regard, the available literature lacks any reference framework; hence this aspect needs to be elucidated by further experiments. In summary, despite the adaptive response that was triggered by the longterm running, the ovary was not protected by the age-related increase of the MG-derived molecular damage, and this was 
likely due to the uncoupled feedback evoked by the exercise regimen on GLO1 and GLO2 expression patterns. Very interestingly, the MG-dependent protein damage appeared to be strongly diminished by the shorter duration of the running programme, maybe as a result of the less severe workload of the shorter training period, yet no indication of enhanced glyoxalase-mediated MG removal was observed following the short-term exercise treatment. These peculiar results seem to confirm that the exercise-induced modulation of the glycative molecular damage in the ovary of mice undergoing the transition from adult to middle age was not driven by the control of the glyoxalase expression, thus providing further indirect clues that changes in the endogenous production rate of MG and/or in the elimination of glycated proteins may participate in the regulatory processes activated in mouse ovaries by a regular exercise programme initiated at the onset of the middle age.

Last but not least, our experiments revealed that the age-associated reduction of the AMH level was partially reversed by the exercise treatment after four months of treadmill running, and this was strongly suggestive that, despite the already compromised ovarian environment, which was maybe dependent on the advanced age at which the exercise training was initiated, a long-term exercise programme is still able to activate positive adaptive changes in the ovarian biochemical milieu which are possibly linked to the improvement of ovarian function and reserve. Obviously, whether slight yet significant exercise-induced elevations of AMH level may trigger any improvement in the ovarian function remains to be established.

\section{Conclusions}

Our results revealed that a regular and moderate running regimen can be helpful to limit the dysfunction of protective systems against oxidative stress that occur within the ovaries of rodents undergoing reproductive aging. Our findings could be useful to develop lifestyle-based nonpharmacological (co)interventions aimed at reducing the vulnerability of mammal ovaries towards age-dependent redoxbased dysfunctions. It remains to be elucidated whether such biochemical adaptations may improve ovarian physiology by delaying follicle or oocyte aging.

\section{Disclosure}

The funder had no role in study design, data collection and analysis, decision to publish, or preparation of the manuscript.

\section{Competing Interests}

The authors declare that there is no conflict of interests regarding the publication of the paper.

\section{Acknowledgments}

The authors wish to thank Dr. Ilenia Taddei for her valuable assistance in the technical editing of this manuscript. This work was funded by the Foundation of the Cassa di Risparmio dell'Aquila (CARISPAQ).

\section{References}

[1] E. R. Te Velde and P. L. Pearson, "The variability of female reproductive ageing," Human Reproduction Update, vol. 8, no. 2, pp. 141-154, 2002.

[2] C. Tatone, F. Amicarelli, M. C. Carbone et al., "Cellular and molecular aspects of ovarian follicle ageing," Human Reproduction Update, vol. 14, no. 2, pp. 131-142, 2008.

[3] S. M. Briley, S. Jasti, J. M. McCracken et al., "Reproductive ageassociated fibrosis in the stroma of the mammalian ovary," Reproduction, vol. 152, no. 3, pp. 245-260, 2016.

[4] S. M. Nelson, E. E. Telfer, and R. A. Anderson, "The ageing ovary and uterus: new biological insights," Human Reproduction Update, vol. 19, no. 1, pp. 67-83, 2013.

[5] K. M. Desai, T. Chang, H. Wang et al., "Oxidative stress and aging: is methylglyoxal the hidden enemy?" Canadian Journal of Physiology and Pharmacology, vol. 88, no. 3, pp. 273-284, 2010.

[6] N. Rabbani and P. J. Thornalley, "Dicarbonyl stress in cell and tissue dysfunction contributing to ageing and disease," Biochemical and Biophysical Research Communications, vol. 458, no. 2, pp. 221-226, 2015.

[7] M. C. Carbone, C. Tatone, S. Delle Monache et al., "Antioxidant enzymatic defences in human follicular fluid: characterization and age-dependent changes," Molecular Human Reproduction, vol. 9, no. 11, pp. 639-643, 2003.

[8] C. Tatone, M. C. Carbone, S. Falone et al., "Age-dependent changes in the expression of superoxide dismutases and catalase are associated with ultrastructural modifications in human granulosa cells," Molecular Human Reproduction, vol. 12, no. 11, pp. 655-660, 2006.

[9] J. Lim and U. Luderer, "Oxidative damage increases and antioxidant gene expression decreases with aging in the mouse ovary," Biology of Reproduction, vol. 84, no. 4, pp. 775-782, 2011.

[10] C. Tatone and F. Amicarelli, "The aging ovary-the poor granulosa cells," Fertility and Sterility, vol. 99, no. 1, pp. 12-17, 2013.

[11] C. Tatone, U. Eichenlaub-Ritter, and F. Amicarelli, "Dicarbonyl stress and glyoxalases in ovarian function," Biochemical Society Transactions, vol. 42, no. 2, pp. 433-438, 2014.

[12] J. Lim, B. N. Nakamura, I. Mohar, T. J. Kavanagh, and U. Luderer, "Glutamate cysteine ligase modifier subunit (Gclm) null mice have increased ovarian oxidative stress and accelerated agerelated ovarian failure," Endocrinology, vol. 156, no. 9, pp. 33293343, 2015.

[13] A. R. Hipkiss, "Energy metabolism, altered proteins, sirtuins and ageing: converging mechanisms?" Biogerontology, vol. 9, no. 1, pp. 49-55, 2008.

[14] M. Pertynska-Marczewska, E. Diamanti-Kandarakis, J. Zhang, and Z. Merhi, "Advanced glycation end products: a link between metabolic and endothelial dysfunction in polycystic ovary syndrome?" Metabolism: Clinical and Experimental, vol. 64, no. 11, pp. 1564-1573, 2015.

[15] L. D. Homa, L. L. Burger, A. J. Cuttitta, D. E. Michele, and S. M. Moenter, "Voluntary exercise improves estrous cyclicity in prenatally androgenized female mice despite programming decreased voluntary exercise: implications for polycystic ovary syndrome (PCOS)," Endocrinology, vol. 156, no. 12, pp. 46184628, 2015. 
[16] S. Falone, A. D’Alessandro, A. Mirabilio et al., "Late-onset running biphasically improves redox balance, energy- and methylglyoxal-related status, as well as SIRT1 expression in mouse hippocampus," PLOS ONE, vol. 7, no. 10, Article ID e48334, 2012.

[17] S. Falone, A. D’Alessandro, A. Mirabilio et al., "Long term running biphasically improves methylglyoxal-related metabolism, redox homeostasis and neurotrophic support within adult mouse brain cortex," PLoS ONE, vol. 7, no. 2, Article ID e31401, 2012.

[18] G. G. Long, "Apparent mesonephric duct (rete anlage) origin for cysts and proliferative epithelial lesions in the mouse ovary," Toxicologic Pathology, vol. 30, no. 5, pp. 592-598, 2002.

[19] M. E. Kevenaar, M. F. Meerasahib, P. Kramer et al., "Serum anti-Müllerian hormone levels reflect the size of the primordial follicle pool in mice," Endocrinology, vol. 147, no. 7, pp. 32283234, 2006.

[20] J. A. Merriman, P. C. Jennings, E. A. McLaughlin, and K. T. Jones, "Effect of aging on superovulation efficiency, aneuploidy rates, and sister chromatid cohesion in mice aged up to 15 months," Biology of Reproduction, vol. 86, no. 2, article 49, 2012.

[21] D.-F. Du, X.-L. Li, F. Fang, and M.-R. Du, "Expression of antiMüllerian hormone in letrozole rat model of polycystic ovary syndrome," Gynecological Endocrinology, vol. 30, no. 12, pp. 885-889, 2014.

[22] W. L. Ledger, "Clinical utility of measurement of anti-müllerian hormone in reproductive endocrinology," Journal of Clinical Endocrinology \& Metabolism, vol. 95, no. 12, pp. 5144-5154, 2010.

[23] S. Di Loreto, S. Falone, A. D’Alessandro et al., "Regular and moderate exercise initiated in middle age prevents age-related amyloidogenesis and preserves synaptic and neuroprotective signaling in mouse brain cortex," Experimental Gerontology, vol. 57, pp. 57-65, 2014.

[24] P. Fernando, A. Bonen, and L. Hoffman-Goetz, "Predicting submaximal oxygen consumption during treadmill running in mice," Canadian Journal of Physiology and Pharmacology, vol. 71, no. 10-11, pp. 854-857, 1993.

[25] M. Szczodry, J. Zhang, C. Lim et al., "Treadmill running exercise results in the presence of numerous myofibroblasts in mouse patellar tendons," Journal of Orthopaedic Research, vol. 27, no. 10, pp. 1373-1378, 2009.

[26] A. A. de Almeida, S. Gomes da Silva, J. Fernandes et al., "Differential effects of exercise intensities in hippocampal BDNF, inflammatory cytokines and cell proliferation in rats during the postnatal brain development," Neuroscience Letters, vol. 553, pp. $1-6,2013$.

[27] M. M. Bradford, "A rapid and sensitive method for the quantitation of microgram quantities of protein utilizing the principle of protein-dye binding," Analytical Biochemistry, vol. 72, no. 1-2, pp. 248-254, 1976.

[28] M. Sun and S. Zigman, "An improved spectrophotometric assay for superoxide dismutase based on epinephrine autoxidation," Analytical Biochemistry, vol. 90, no. 1, pp. 81-89, 1978.

[29] H. Aebi, "[13] Catalase in vitro," Methods in Enzymology, vol. 105, no. C, pp. 121-126, 1984.

[30] D. E. Paglia and W. N. Valentine, "Studies on the quantitative and qualitative characterization of erythrocyte glutathione peroxidase," The Journal of Laboratory and Clinical Medicine, vol. 70, no. 1, pp. 158-169, 1967.

[31] B. Mannervik, A. C. Aronsson, E. Marmstål, and G. Tibbelin, "Glyoxalase I (rat liver)," Methods in Enzymology, vol. 77, pp. 297-301, 1981.
[32] M. K. Guha, D. L. Vander Jagt, and D. J. Creighton, "Diffusiondependent rates for the hydrolysis reaction catalyzed by glyoxalase II from rat erythrocytes," Biochemistry, vol. 27, no. 24, pp. 8818-8822, 1988.

[33] M. A. Baker, G. J. Cerniglia, and A. Zaman, "Microtiter plate assay for the measurement of glutathione and glutathione disulfide in large numbers of biological samples," Analytical Biochemistry, vol. 190, no. 2, pp. 360-365, 1990.

[34] M. M. Jack, J. M. Ryals, and D. E. Wright, "Characterisation of glyoxalase I in a streptozocin-induced mouse model of diabetes with painful and insensate neuropathy," Diabetologia, vol. 54, no. 8, pp. 2174-2182, 2011.

[35] K. J. Livak and T. D. Schmittgen, "Analysis of relative gene expression data using real-time quantitative PCR and the $2^{-\Delta \Delta C_{\mathrm{T}}}$ method," Methods, vol. 25, no. 4, pp. 402-408, 2001.

[36] U. K. Laemmli, "Cleavage of structural proteins during the assembly of the head of bacteriophage T4," Nature, vol. 227, no. 5259, pp. 680-685, 1970.

[37] H. Towbin, T. Staehelin, and J. Gordon, "Electrophoretic transfer of proteins from polyacrylamide gels to nitrocellulose sheets: procedure and some applications," Proceedings of the National Academy of Sciences of the United States of America, vol. 76, no. 9, pp. 4350-4354, 1979.

[38] R. L. Levine, J. A. Williams, E. R. Stadtman, and E. Shacter, "Carbonyl assays for determination of oxidatively modified proteins," Methods in Enzymology, vol. 233, pp. 346-357, 1994.

[39] J. Hellemans, G. Mortier, A. De Paepe, F. Speleman, and J. Vandesompele, "qBase relative quantification framework and software for management and automated analysis of real-time quantitative PCR data," Genome Biology, vol. 8, no. 2, p. R19, 2007.

[40] A. Bonfigli, S. Colafarina, S. Falone, C. Di Giulio, C. Di Ilio, and F. Amicarelli, "High levels of antioxidant enzymatic defence assure good protection against hypoxic stress in spontaneously diabetic rats," International Journal of Biochemistry and Cell Biology, vol. 38, no. 12, pp. 2196-2208, 2006.

[41] E.-M. Park, N. Ramnath, G. Y. Yang et al., "High superoxide dismutase and low glutathione peroxidase activities in red blood cells predict susceptibility of lung cancer patients to radiation pneumonitis," Free Radical Biology \& Medicine, vol. 42, no. 2, pp. 280-287, 2007.

[42] National Institute of Health, NIH publication no. 11-7737, October 2011, https://www.nia.nih.gov/sites/default/files/global_ health_and_aging.pdf.

[43] United Nations, "World Population Aging 2013," New York, 2013.

[44] C. E. Finch, "The menopause and aging, a comparative perspective," The Journal of Steroid Biochemistry and Molecular Biology, vol. 142, pp. 132-141, 2014.

[45] S. M. Somani, K. Husain, and E. C. Schlorff, "Response of antioxidant system to physical and chemical stress," in Oxidant, Antioxidants and Free Radicals, S. I. Baskin and H. Salem, Eds., pp. 125-141, Taylor \& Francis, London, UK, 1997.

[46] S. K. Powers and C. K. Sen, "Physiological antioxidants and exercise training," in Handbook of Oxidants and Antioxidants in Exercise, C. K. Sen, L. Packer, and O. Hanninen, Eds., Elsevier Science B.V., Amsterdam, The Netherlands, 2000.

[47] S. Das, R. Chattopadhyay, S. Ghosh et al., "Reactive oxygen species level in follicular fluid-embryo quality marker in IVF?" Human Reproduction, vol. 21, no. 9, pp. 2403-2407, 2006. 
[48] Z. Wiener-Megnazi, L. Vardi, A. Lissak et al., "Oxidative stress indices in follicular fluid as measured by the thermochemiluminescence assay correlate with outcome parameters in in vitro fertilization," Fertility and Sterility, vol. 82, no. 3, pp. 1171-1176, 2004.

[49] F. A. V. Seixas, M. R. da Silva, M. T. Murakami, P. Tosqui, and M. F. Colombo, "The water effect on the kinetics of the bovine liver catalase," Protein \& Peptide Letters, vol. 18, no. 9, pp. 879-885, 2011.

[50] R. Masella, R. Di Benedetto, R. Varì, C. Filesi, and C. Giovannini, "Novel mechanisms of natural antioxidant compounds in biological systems: involvement of glutathione and glutathionerelated enzymes," The Journal of Nutritional Biochemistry, vol. 16, no. 10, pp. 577-586, 2005.

[51] Q. Gu, B. Wang, X.-F. Zhang, Y.-P. Ma, J.-D. Liu, and X.-Z. Wang, "Contribution of receptor for advanced glycation end products to vasculature-protecting effects of exercise training in aged rats," European Journal of Pharmacology, vol. 741, pp. 186-194, 2014.

[52] J. Liu, M. Liu, X. Ye et al., "Delay in oocyte aging in mice by the antioxidant N-acetyl-l-cysteine (NAC)," Human Reproduction, vol. 27, no. 5, pp. 1411-1420, 2012.

[53] A. Ahangarpour, Z. Lamoochi, H. Fathi Moghaddam, and S. M. Mansouri, "Effects of Portulaca oleracea ethanolic extract on reproductive system of aging female mice," International Journal of Reproductive BioMedicine, vol. 14, no. 3, pp. 205-212, 2016.

[54] M. Mahmoodi, M. Soleimani Mehranjani, S. M. A. Shariatzadeh, H. Eimani, and A. Shahverdi, "N-acetylcysteine improves function and follicular survival in mice ovarian grafts through inhibition of oxidative stress," Reproductive BioMedicine Online, vol. 30, no. 1, pp. 101-110, 2015.

[55] D. Bae, J. B. Park, J. U. Yang, and T. H. Lee, "Purification and characterization of glyoxylase II from bovine liver," Korean Journal of Biochemistry, vol. 27, pp. 99-103, 1995.

[56] S. Falone, S. Jr Santini, S. di Loreto et al., "Improved mitochondrial and methylglyoxal-related metabolisms support hyperproliferation induced by $50 \mathrm{~Hz}$ magnetic field in neuroblastoma cells," Journal of Cellular Physiology, vol. 231, no. 9, pp. 20142025, 2016. 


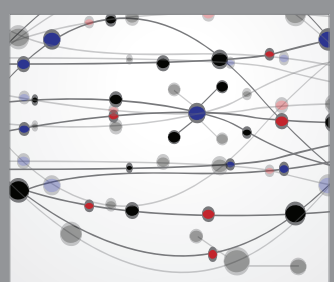

The Scientific World Journal
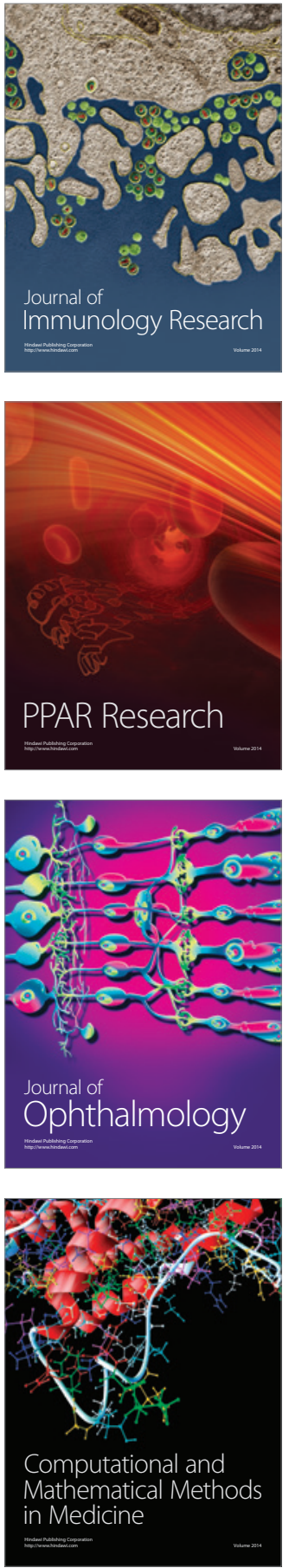

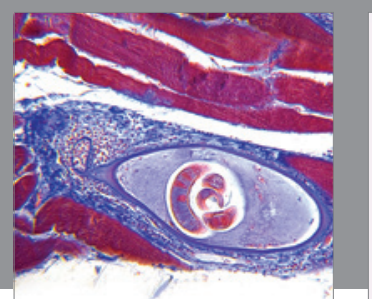

Gastroenterology Research and Practice

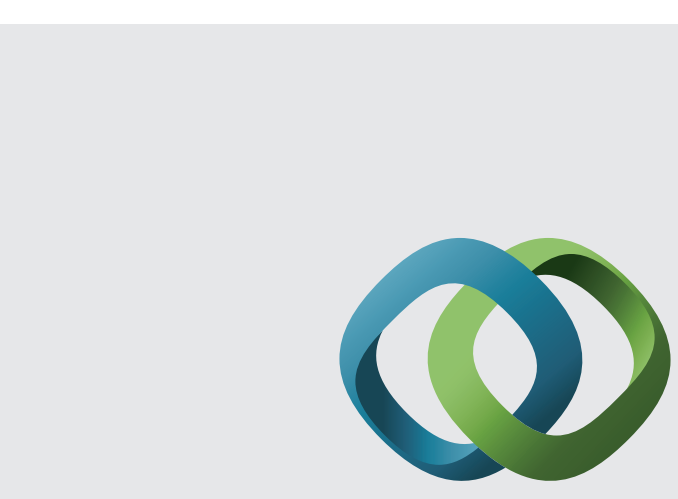

\section{Hindawi}

Submit your manuscripts at

http://www.hindawi.com
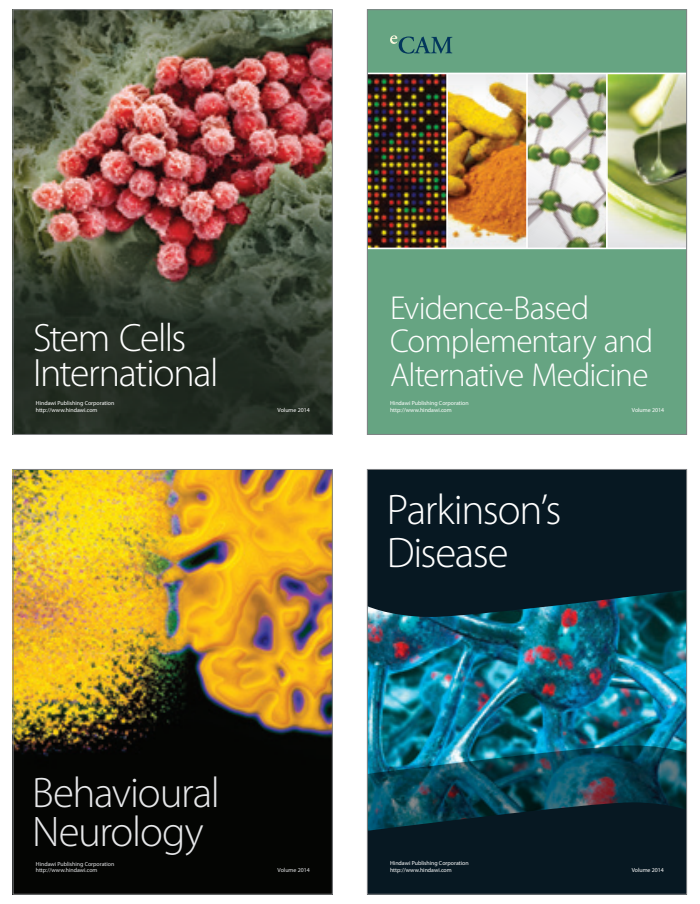
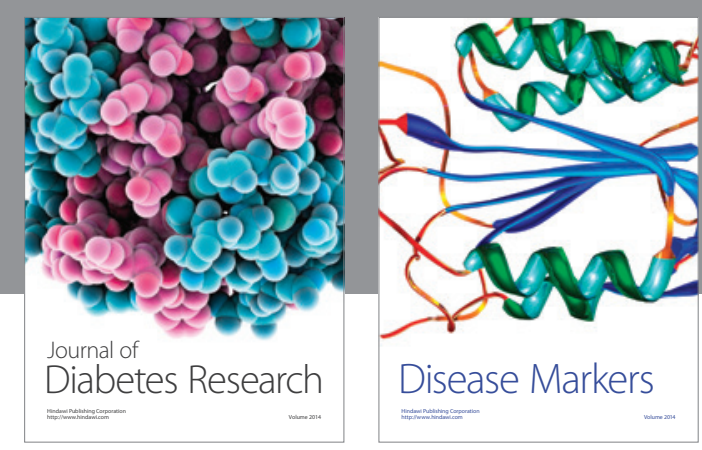

Disease Markers
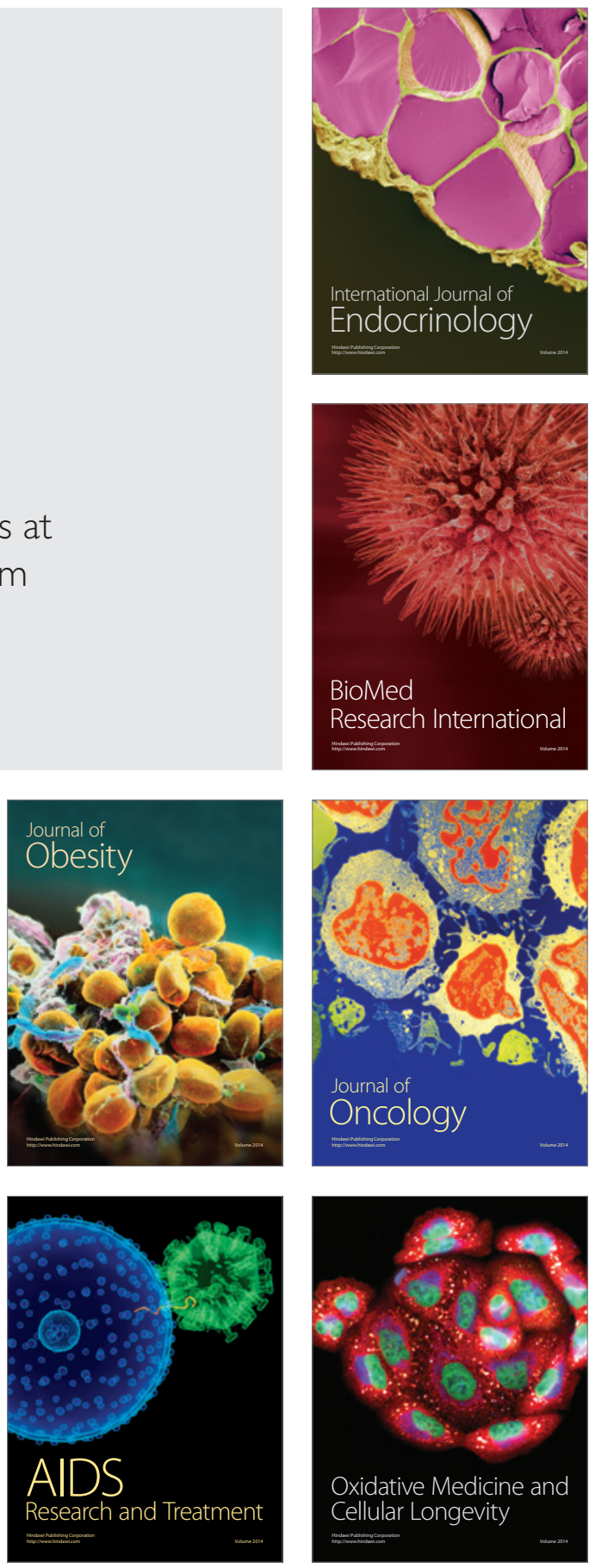